\title{
Prognostic Value of Axillary Nodal Ratio after Neoadjuvant Chemotherapy of Doxorubicin/Cyclophosphamide Followed by Docetaxel in Breast Cancer: A Multicenter Retrospective Cohort Study
}

\section{Se Hyun Kim, MD \\ Kyung Hae Jung, MD² \\ Tae-Yong Kim, MD \\ Seock-Ah Im, MD ${ }^{3}$ \\ In Sil Choi, $\mathrm{MD}^{4}$ \\ Yee Soo Chae, MD \\ Sun Kyung Baek, MD \\ Seok Yun Kang, $\mathrm{MD}^{7}$ \\ Sarah Park, MD \\ In Hae Park, MD9 \\ Keun Seok Lee, MD 9 \\ Yoon Ji Choi, MD ${ }^{10}$ \\ Soohyeon Lee, MD ${ }^{11}$ \\ Joo Hyuk Sohn, MD ${ }^{11}$ \\ Yeon-Hee Park, MD'2 \\ Young-Hyuck Im, MD ${ }^{12}$ \\ Jin-Hee Ahn, MD² \\ Sung-Bae Kim, MD2 \\ Jee Hyun Kim, MD'}

${ }^{*} A$ list author's affiliations appears at the end of the paper.
Correspondence: Jee Hyun Kim, MD

Division of Hematology and Medical Oncology, Department of Internal Medicine,

Seoul National University Bundang Hospital, Seoul National University College of Medicine, 82 Gumi-ro 173beon-gil, Bundang-gu,

Seongnam 13620, Korea

Tel: 82-31-787-7022

Fax: 82-31-787-4098

E-mail: jhkimmd@snu.ac.kr

Received December 8, 2015

Accepted March 3, 2016

Published Online March 23, 2016

*Se Hyun Kim and Kyung Hae Jung contributed equally to this work.

\section{Purpose}

The purpose of this study is to investigate the prognostic value of lymph node (LN) ratio (LNR) in patients with breast cancer after neoadjuvant chemotherapy.

\section{Materials and Methods}

This retrospective analysis is based on the data of 814 patients with stage II/III breast cancer treated with four cycles of doxorubicin/cyclophosphamide followed by four cycles of docetaxel before surgery. We evaluated the clinical significance of LNR (3 categories: low 0-0.20 vs. intermediate 0.21-0.65 vs. high 0.66-1.00) using a Cox proportional regression model.

Results

A total of 799 patients underwent breast surgery. Pathologic complete response (pCR, ypTO/isN0) was achieved in 129 patients (16.1\%) (hormone receptor [HR] +/human epidermal growth factor receptor 2 [HER2] -, 34/373 [9.1\%]; HER2+, 45/210 [21.4\%]; triple negative breast cancer, 50/216 [23.1\%]). The mean numbers of involved LN and retrieved LN were 2.70 (range, 0 to 42) and 13.98 (range, 1 to 64 ), respectively. The mean LNR was 0.17 (low, 574 [71.8\%]; intermediate, 170 [21.3\%]; high, 55 [6.9\%]). In univariate analysis, LNR showed significant association with a worse relapse-free survival (3-year relapse-free survival rate $84.8 \%$ in low vs. $66.2 \%$ in intermediate vs. $54.3 \%$ in high; $p<0.001$, log-rank test). In multivariate analysis, LNR did not show significant association with recurrence after adjusting for other clinical factors (age, histologic grade, subtype, ypT stage, ypN stage, lymphatic or vascular invasion, and pCR). In subgroup analysis, the LNR system had good prognostic value in HR+/HER2- subtype.

\section{Conclusion}

LNR is not superior to ypN stage in predicting clinical outcome of breast cancer after neoadjuvant chemotherapy. However, the prognostic value of the LNR system in HR+/HER2patients is notable and worthy of further investigation.

\section{Key words}

Lymph nodes, Lymph node excision, Neoadjuvant therapy, Breast neoplasms, Prognosis 


\section{Introduction}

Neoadjuvant chemotherapy (NCT) is a treatment option for patients with operable breast cancer, who are candidates for adjuvant chemotherapy. The equivalency of NCT and adjuvant chemotherapy with regard to disease-free survival and overall survival (OS) was demonstrated in large randomized trials [1-3]. NCT has a number of advantages compared to adjuvant chemotherapy, including downsizing the tumor to increase the likelihood of breast conservation, providing in vivo chemosensitivity to oncologists, and enabling rapid assessment of the efficacy of new therapeutic agents. Importantly, the pathologic complete response (pCR) after NCT has a strong association with improved survival in patients with aggressive breast cancer subtypes [4].

The best established predictor of survival is the absolute number of involved lymph nodes (LNs) in the axilla following NCT, which is in accordance with the ypN stage of the American Joint Committee on Cancer (AJCC) staging system [2,5-8]. However, based on previous observations, NCT may change the histological environment of the axillary area and reduce the number of retrieved axillary LNs $[9,10]$. As a result, the ypN stage can be underestimated, thereby resulting in inadequate treatment. Many studies with adjuvant chemotherapy suggested that the lymph node ratio (LNR), the ratio of the number of involved LNs to the total number of resected LNs, may be a superior prognostic factor than the pN stage [11-15]. Ahn et al. [14] concluded that LNR may be superior to the $\mathrm{pN}$ stage as a prognostic factor and can be used in identification of patient subgroups that may benefit from adjuvant radiotherapy. Dings et al. [15] reported that LNR can differentiate populations with poor prognosis within the same $\mathrm{pN}$ stage. In a recent meta-analysis, Liu et al. [16] reported that LNR was a prognostic predictor for breast cancer. After analysis of the studies that used 0.2 and 0.65 as the cut-off, the authors confirmed that there was a dose-response relationship between LNR and OS, diseasefree survival, breast cancer specific survival, and mortality. However, there are limited data and conflicting results with regard to the prognostic significance of LNR after NCT $[17,18]$.

In this study, we investigated the prognostic value of LNR in patients treated with NCT and compared LNR with the traditional ypN stage of the AJCC in predicting disease recurrence.

\section{Materials and Methods}

\section{Study population}

This study included 814 consecutive patients from 13 academic hospitals of the Korean Cancer Study Group. They were pathologically diagnosed with stage II/ III breast cancer and received treatment with four cycles of doxorubicin/ cyclophosphamide (AC), followed by four cycles of docetaxel (DOC) as NCT within a study period from June 2009 to December 2012. Among the 814 patients, 799 had undergone surgery and were analyzed. Clinical data were obtained from the electronic medical records of each hospital. The data included demographics, past medical history, pathologic information, radiologic findings, NCT, adjuvant treatment, recurrence, and survival. Male breast cancer, inflammatory breast cancer, recurrent or metastatic breast cancer, any treatment before NCT, and other coexisting malignancies were excluded. This study was approved by the Institutional Review Board at Seoul National University Bundang Hospital (Seongnam, Korea) and participating institutions.

\section{Treatment and tumor assessment}

All patients were treated with four cycles of AC (doxorubicin $60 \mathrm{mg} / \mathrm{m}^{2}$ on day 1 and cyclophosphamide $600 \mathrm{mg} / \mathrm{m}^{2}$ on day 1 every 21 days) followed by four cycles of DOC (75 mg/ $\mathrm{m}^{2}$ on day 1 every 21 days). In patients with overexpression or amplification of human epidermal growth factor receptor 2 (HER2), trastuzumab was incorporated into the adjuvant treatment and administered for 1 year after surgery. The clinical response to NCT was evaluated every four cycles, using ultrasound or magnetic resonance imaging in accordance with the Response Evaluation Criteria in Solid Tumors (RECIST) ver. 1.1.

Pathological assessment was performed with core biopsy samples or surgical specimens of primary tumors by a pathologist at each institution. Immunohistochemistry (IHC) was performed for the estrogen receptor, progesterone receptor, and HER2. In patients with HER2 IHC score of 2+, HER2 amplification status was confirmed by fluorescent in situ hybridization or silver in situ hybridization. pCR was defined as ypT0N0 (absence of invasive cancer and in situ cancer in the breast and axillary nodes) or ypT0/isN0 (absence of invasive cancer in the breast and axillary nodes). From the pathological report of the surgical specimen, LNR was calculated as the ratio of the number of involved LNs to the number of total retrieved LNs. LNR was categorized according to three groups based on previous findings: low (LNR, 0 to 0.20$)$, intermediate $(0.21$ to 0.65$)$, and high ( 0.66 to 1.00) [12-14]. 


\section{Statistical analysis}

Data were described as frequencies (\%), or means and medians (range). An independent two-sample t test was used for analysis of continuous variables, and chi-square test for categorical variables. The primary end point was relapse-free survival (RFS), calculated from the start of NCT to recurrence of breast cancer. Patients who were relapse free at the last contact were censored at the last follow-up date. The secondary end points were the rate of distant recurrence and OS. OS was determined from the initial NCT to death from any cause. The Kaplan-Meier method was used for estimation of survival outcomes and the log-rank test was used for determination of differences between the groups. Cox proportional hazard regression analysis was used for estimation of hazard ratios and 95\% confidence intervals (CIs). Variables with statistical significance $(\mathrm{p}<0.05)$ in univariate analysis were included as covariates in multivariate analysis. A value of $\mathrm{p}<0.05$ was considered to indicate statistical significance, and all resulting p-values were two sided. All statistical procedures were performed using SPSS ver. 18.0 (SPSS Inc., Chicago, IL).

\section{Results}

\section{Patient characteristics}

A total of 799 patients were included in our study (Table 1). The median age was 45 years (range, 16 to 74 years), and the majority (88.1\%) underwent axillary LN dissection. Before surgery, 687 patients $(85.9 \%)$ had undergone fine needle aspiration biopsy or sentinel LN biopsy $(\mathrm{n}=19,2.4 \%)$ for axillary staging. Almost all patients $(741 / 799,92.8 \%)$ received eight cycles of NCT. The pCR rate and LN status according to subtype of breast is shown in Table 2. The overall pCR (T0/ isN0) rate was 16.1\% (129/799); 9.1\% (34/373) in HR+/HER2- patients, 21.4\% (45/210) in HER2+ patients, and 23.1\% (50/216) in triple negative breast cancer (TNBC) patients. Among the pathologically confirmed $\mathrm{LN}+$ patients $(\mathrm{n}=622)$, pathologic axillary $\mathrm{LN}$ clearance (ypN0) was achieved in 243 patients (39.1\%). The median numbers of involved LNs and retrieved LNs were one (range, 0 to 42 ) and 13 (range, 1 to 64 ), respectively. Most patients $(759 / 799,95 \%)$ had more than four retrieved LNs after surgery (Supplementary Table 1). The median and mean numbers of LNR were 0.07 and 0.17 , respectively. When using the LNR classification, 574 patients (71.8\%) were categorized as low (LNR, 0 to 0.20$), 170(21.3 \%)$ as intermediate $(0.21$ to 0.65$)$, and $55(6.9 \%)$ as high (0.66 to
Table 1. Baseline characteristics of patients

\begin{tabular}{|c|c|}
\hline Characteristic & No. $(\%)$ \\
\hline \multicolumn{2}{|l|}{ Age (yr) } \\
\hline Median (range) & $45(16-74)$ \\
\hline \multicolumn{2}{|l|}{ ECOG PS } \\
\hline 0 & $470(58.8)$ \\
\hline 1 & $327(40.9)$ \\
\hline 2 & $2(0.3)$ \\
\hline \multicolumn{2}{|l|}{ Histology } \\
\hline Ductal & $762(95.4)$ \\
\hline Lobular & $15(1.9)$ \\
\hline Others & $22(2.7)$ \\
\hline \multicolumn{2}{|l|}{ Histologic grade } \\
\hline 1 & $32(4)$ \\
\hline 2 & $379(47.4)$ \\
\hline 3 & $304(38)$ \\
\hline Unknown & $84(10.5)$ \\
\hline \multicolumn{2}{|c|}{ Tumor size at diagnosis $(\mathrm{cm})$} \\
\hline Mean \pm SD & $4.3 \pm 2.36$ \\
\hline Median (range) & $3.8(0.8-20)$ \\
\hline \multicolumn{2}{|l|}{ cT stage } \\
\hline 0 & $6(0.8)$ \\
\hline 1 & $54(6.8)$ \\
\hline 2 & $440(55.1)$ \\
\hline 3 & $238(29.8)$ \\
\hline 4 & $61(7.6)$ \\
\hline \multicolumn{2}{|l|}{ cN stage } \\
\hline 0 & $42(5.3)$ \\
\hline 1 & $379(47.4)$ \\
\hline 2 & $238(29.8)$ \\
\hline 3 & $140(17.5)$ \\
\hline \multicolumn{2}{|l|}{ ER receptor } \\
\hline Positive & $454(56.8)$ \\
\hline Negative & $345(43.2)$ \\
\hline \multicolumn{2}{|l|}{ PR receptor } \\
\hline Positive & $384(48.1)$ \\
\hline Negative & $415(51.9)$ \\
\hline \multicolumn{2}{|l|}{ Subtype } \\
\hline HR+/HER2- & 373 (46.7) \\
\hline HER2+ & $210(26.3)$ \\
\hline TNBC & $216(27)$ \\
\hline
\end{tabular}

ECOG, Eastern Cooperative Oncology Group; PS, performance status; $\mathrm{SD}$, standard deviation; ER, estrogen receptor; PR, progesterone receptor; HR, hormone receptor; HER2, human epidermal growth factor receptor 2; $\mathrm{TNBC}$, triple negative breast cancer. 
Table 2. Pathological response and lymph node status after NCT

\begin{tabular}{|c|c|c|c|c|c|}
\hline Variable & Total & HR+/HER2- & HER2+ & TNBC & p-value \\
\hline \multicolumn{6}{|c|}{ No. of lymph nodes } \\
\hline Involved & $2.70(0-42)$ & $3.12(0-41)$ & $2.25(0-30)$ & $2.42(0-42)$ & 0.083 \\
\hline Retrieved & $13.98(1-64)$ & $14.20(1-47)$ & $13.62(1-38)$ & $13.96(1-64)$ & 0.737 \\
\hline \multicolumn{6}{|c|}{ ypN stage by AJCC } \\
\hline 0 & 335 (41.9) & $106(28.4)$ & $100(47.6)$ & $129(59.7)$ & $<0.001$ \\
\hline 1 & $286(35.8)$ & $166(44.5)$ & $71(33.8)$ & $49(22.7)$ & \\
\hline 2 & $116(14.5)$ & $71(19)$ & $27(12.9)$ & $18(8.3)$ & \\
\hline 3 & $62(7.8)$ & $30(8)$ & $12(5.7)$ & $20(9.3)$ & \\
\hline \multicolumn{6}{|c|}{ Lymph node ratio } \\
\hline Low & $574(71.8)$ & $236(63.4)$ & $163(77.6)$ & $174(80.6)$ & $<0.001$ \\
\hline Intermediate & $170(21.3)$ & $108(29)$ & $34(16.2)$ & $28(16.5)$ & \\
\hline High & $55(6.9)$ & $28(7.5)$ & $13(6.2)$ & $14(6.5)$ & \\
\hline \multicolumn{6}{|c|}{ pCR (ypT0/isN0) } \\
\hline Yes & $129(16.1)$ & $34(9.1)$ & $45(21.4)$ & $50(23.1)$ & $<0.001$ \\
\hline No & $670(83.9)$ & 339 (90.9) & 165 (78.6) & $166(76.9)$ & \\
\hline
\end{tabular}

Values are presented as mean (range) or number (\%). NCT, neoadjuvant chemotherapy; HR, hormone receptor; HER2, human epidermal growth factor receptor 2; TNBC, triple negative breast cancer; AJCC, American Joint Committee on Cancer; pCR, pathologic complete response.

A
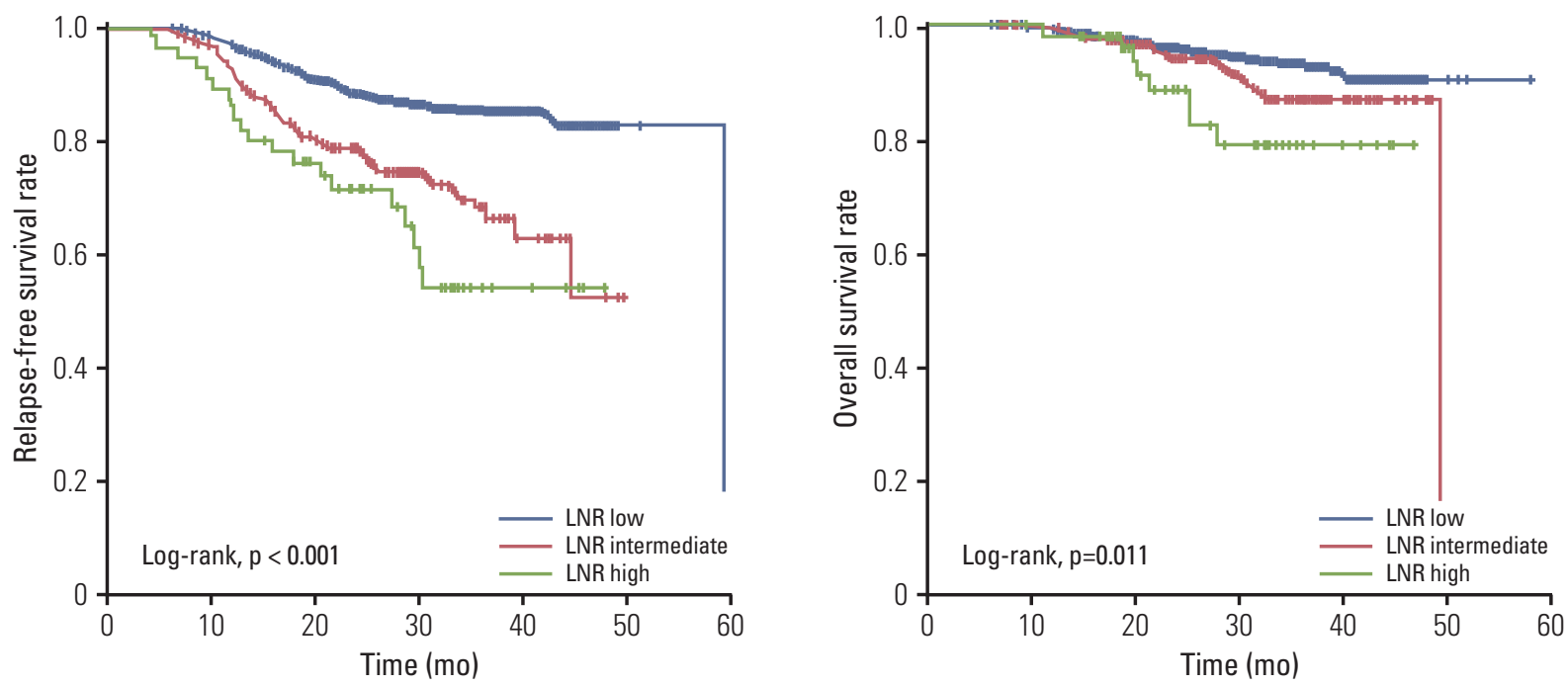

Fig. 1. Survival of patients according to lymph node ratio (LNR) status: relapse-free survival (A) and overall survival (B).

1.00). Patients with HR+/HER2- subtype had a significantly lower rate of ypN0 stage compared with other subtypes ( $28.4 \%$ vs. $47.6 \%$ in HER2 and $59.7 \%$ in TNBC, $\mathrm{p}<0.001)$. Approximately two-thirds of patients with HR+ / HER2subtype were categorized as the low LNR group ( $63.4 \%$ in HR+/HER2- vs. $77.6 \%$ in HER2 and $80.6 \%$ in TNBC, $\mathrm{p}<0.001)$.

\section{Survival and relapse according to axillary LN status}

RFS and OS were evaluated with a median follow-up period of 31.0 months. With the LNR classification, the 3-year RFS rates were $84.8 \%, 66.2 \%$, and $54.3 \%$ in low, intermediate, and high groups, respectively $(\mathrm{p}<0.001)$ (Fig. 1A). The estimated 3-year OS rates were $92.7 \%, 87.1 \%$, and $79.3 \%$ in the 
low, intermediate, and high groups, respectively $(\mathrm{p}=0.011)$ (Fig. 1B). Using the ypN-stage classification, the 3-year RFS rates were $88.1 \%, 80.8 \%, 61.8 \%$, and $48.1 \%$ in ypN0, ypN1, ypN2, and ypN3 stages, respectively $(\mathrm{p}<0.001)$. Probabilities of 3-year OS were $94.6 \%, 91.5 \%, 87.3 \%$, and $69.6 \%$ in ypN0, ypN1, ypN2, and ypN3 stage, respectively $(\mathrm{p}<0.001)$.

To examine the pattern of relapse in accordance with LNR status, disease recurrence was classified as locoregional, distant only, and locoregional with distant relapse (Supplementary Table 2). The increase of LNR showed significant association with the distant failure rate $(9.5 \%$ in low group vs. $18.2 \%$ in intermediate group vs. $27.3 \%$ in high group, chi-square $\mathrm{p}<0.001$ ).

\section{Survival analysis}

Among the variables analyzed in the univariate Cox model, pCR status, histologic grade, subtype by IHC, ypT stage, ypN stage, lymphovascular invasion (LVI), and LNR classification showed significant association with RFS in all patients. The LNR classification was not an independent predictor for RFS in the multivariate model adjusted for age, pCR status, histologic grade, subtype by IHC, ypT stage,
ypN stage, and LVI ( $p=0.954)$ (Table 3). To exclude the possibility of any confounding effect, we constructed two multivariate models with either ypN stage or LNR classification. Both ypN stage and LNR classification were independent predictors for RFS in each model. By calculating the log likelihood chi-squares of each model, we were able to compare the fit of each model (higher value indicates a more predictive model). However, the values of log likelihood chi-squares of both models were similar (Supplementary Table 3).

\section{Prognostic value of LNR according to subtype by IHC}

In HR+ / HER2- patients, a statistically significant difference in RFS was observed in accordance with LNR status during the follow-up period (LNR-low vs. LNR-intermediate, $p=0.045$; LNR-low vs. LNR-high, $p=0.001$; LNR-intermediate vs. LNR-high, $\mathrm{p}=0.027$ ) (Fig. 2). In HER2+ and TNBC patients, LNR-low showed association with better RFS than LNR-intermediate or LNR-high (LNR-low vs. LNR-intermediate, $\mathrm{p}<0.001$ in HER2+ and $\mathrm{p}<0.001$ in TNBC; LNR-low vs. LNR-high, $\mathrm{p}=0.001$ in HER2+ and $\mathrm{p}<0.001$ in TNBC; logrank test) (Fig. 2). However, no significant difference in RFS

Table 3. Multivariate analysis for relapse-free survival of patients

\begin{tabular}{|c|c|c|c|}
\hline Variable & Hazard ratio & $95 \% \mathrm{CI}$ & p-value \\
\hline $\operatorname{Age}(<50$ yr vs. $>50$ yr $)$ & 0.77 & $0.53-1.11$ & 0.157 \\
\hline Histologic grade 1 & 1 & & 0.001 \\
\hline Histologic grade 2 & 1.2 & $0.37-3.92$ & \\
\hline Histologic grade 3 & 2.59 & $0.78-8.57$ & \\
\hline Histologic grade NA & 1.27 & $0.33-4.94$ & \\
\hline Subtype: HR+/HER2- & 1 & & $<0.001$ \\
\hline Subtype: HER2+ & 1.69 & $1.02-2.79$ & \\
\hline Subtype: TNBC & 4.97 & $3.24-7.61$ & \\
\hline pCR (ypT0/isN0) vs. non-pCR & 0.26 & $0.08-0.86$ & 0.027 \\
\hline ypT stage 0 & 1 & & $<0.001$ \\
\hline ypT stage 1 & 0.85 & $0.39-1.83$ & \\
\hline ypT stage 2 & 1.96 & $0.90-4.24$ & \\
\hline ypT stage 3 & 1.95 & $0.69-3.95$ & \\
\hline ypT stage 4 & 5.93 & $1.63-21.58$ & \\
\hline ypN stage 0 & 1 & & 0.035 \\
\hline ypN stage 1 & 1.72 & $1.04-2.84$ & \\
\hline ypN stage 2 & 2.69 & $1.27-5.71$ & \\
\hline ypN stage 3 & 3.39 & $1.43-8.01$ & \\
\hline Lymphovascular invasion (yes vs. no) & 1.5 & $1.02-2.22$ & 0.040 \\
\hline LNR low $(0-0.20)$ & 1 & & 0.954 \\
\hline LNR intermediate $(0.21-0.65)$ & 1.01 & $0.55-1.86$ & \\
\hline LNR high (0.66-1.00) & 1.12 & $0.48-2.59$ & \\
\hline
\end{tabular}

CI, confidence interval; NA, not available; HR, hormone receptor; HER2, human epidermal growth factor receptor 2; TNBC, triple negative breast cancer; pCR, pathologic complete response; LNR, lymph node ratio. 
A
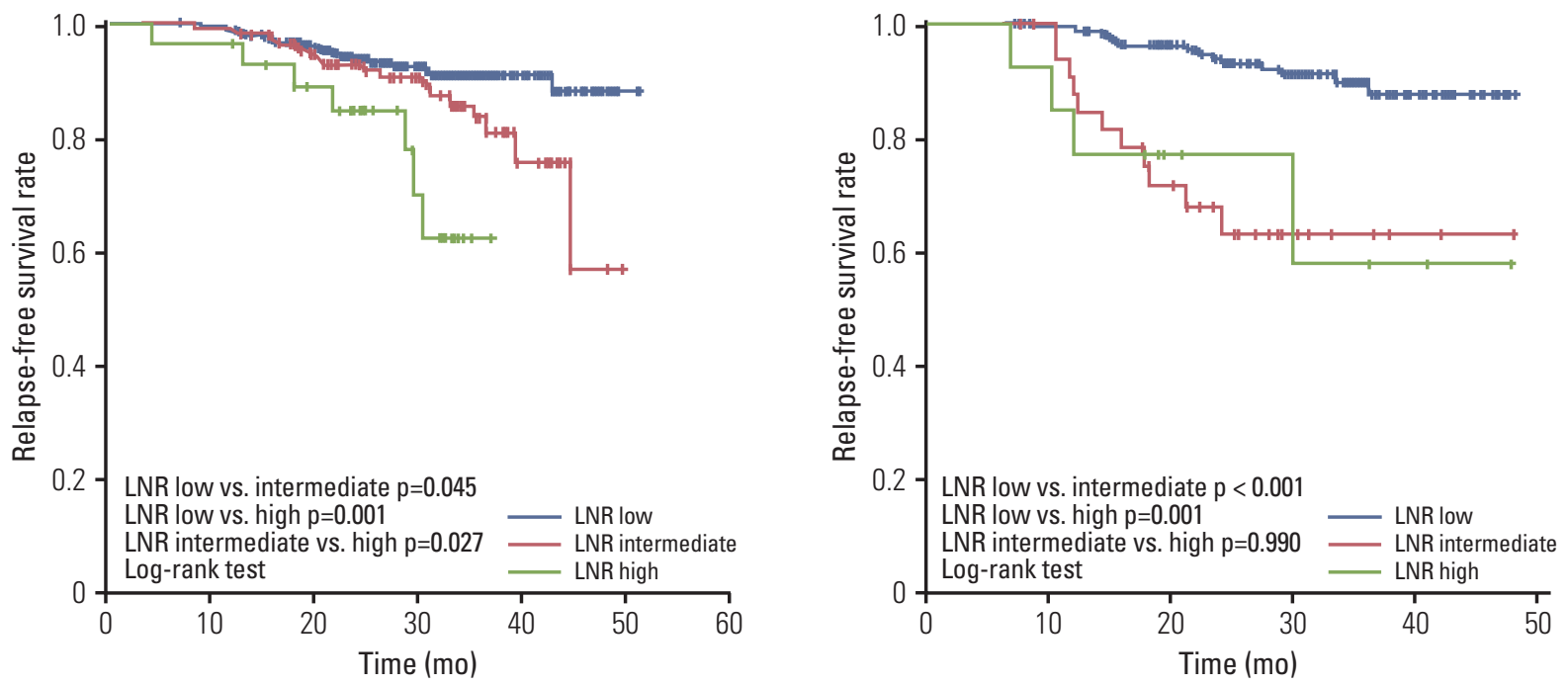

C

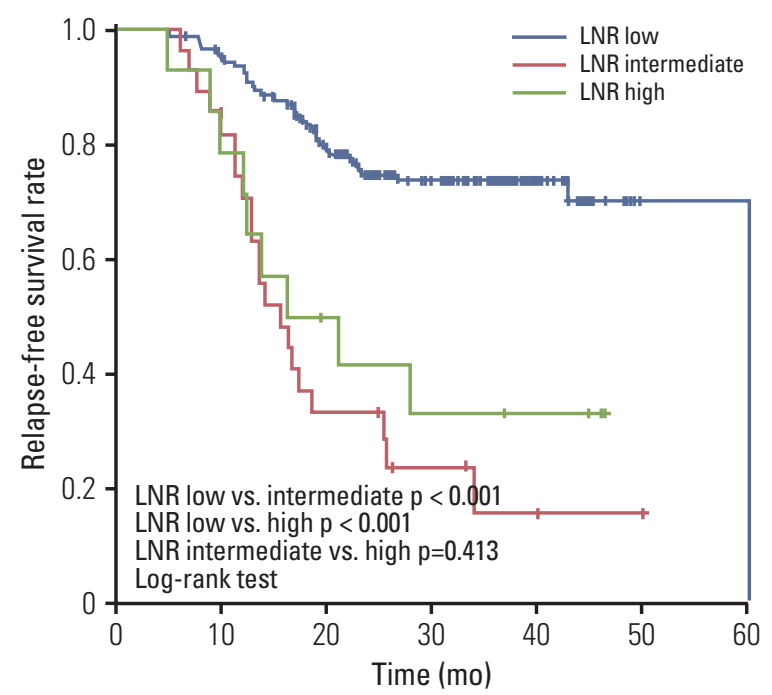

Fig. 2. Relapse-free survival of patients according to lymph node ratio (LNR) status and subtype: hormone receptor +/human epidermal growth factor receptor 2 (HER2) - (A), HER2+ (B), and triple negative breast cancer (C).

was observed between LNR-intermediate and LNR-high (LNR-intermediate vs. LNR-high, $\mathrm{p}=0.990$ in HER2 + and $\mathrm{p}=0.413$ in TNBC) (Fig. 2). The LNR classification was not an independent predictor for RFS in the multivariate model in each subtype (Supplementary Table 4).

\section{Discussion}

In this study, we demonstrated that the axillary LN status, either ypN stage or LNR, is of prognostic value in breast cancer patients treated with NCT. Patients with higher LNR had poorer RFS and OS. In comparison of the value of log likelihood chi-squares between two models, the prognostic power of LNR after NCT, using the cut-off points of 0.20 and 0.65 , appears to be similar to that of the current ypN stage in 
our patient cohort. To the best of our knowledge, this study is the largest study to date addressing the prognostic value of LNR in patients uniformly treated with eight courses of sequential anthracycline-taxane-based NCT.

It should be noted that the number of retrieved and examined LNs is influenced by NCT $[9,10]$. In our study, most patients $(92.8 \%)$ completed the preplanned eight cycles of NCT, while only three cycles of NCT were utilized in previous studies [17,18]. In the most recent study, Chen et al. [19] reported a better prognostic value of LNR than ypN stage in patients treated with median three cycles of NCT. The pCR rate of $16.1 \%$ in our study is higher than that of other studies (range, 6.8\% to 10.7\%) reporting that the LNR system is superior to ypN stage $[17,19]$. The longer duration with more effective NCT in the current study may result in a lower number of involved LNs than that of the two previous studies (Supplementary Table 5). The reason for the consistent results with regard to the prognostic significance of LNR in an adjuvant setting compared to the conflicting results in a neoadjuvant setting may be derived in part from less involvement of LNs in studies with NCT. We suppose that there might be an inverse correlation between the efficacy of NCT and prognostic impact of LNR.

We observed a low pCR rate $(9.1 \%)$ and good separation of RFS curves with the LNR system in HR+/HER2- patients. Conversely, a high pCR rate $(21.4 \%$ in HER $2+$ and $23.1 \%$ in TNBC) and overlapping RFS curves were observed in HER2 and TNBC patients. These findings are in line with those of recent studies reporting a strong dependence on the intrinsic subtype for a relationship between $\mathrm{pCR}$ rate and survival $[4,20,21]$. In addition, the prognostic value of the LNR system in HR+/HER2- patients is notable because it could have a role in selection of high-risk patients for adjuvant radiotherapy or prolonged duration of adjuvant endocrine therapy. Tausch et al. [22], who reported that LNR was an additional prognostic factor in patients with one to three involved LNs after mastectomy, suggested LNR as an indicator for postmastectomy radiotherapy, which is still controversial in patients with one to three LNs [23]. Currently, identification of patients who might benefit from extended adjuvant endocrine therapy is not possible. The findings by Sestak et al. [24] suggest that clinical factors, including nodal status (positive vs. negative) and tumor size ( $>2 \mathrm{~cm}$ vs. $<2 \mathrm{~cm}$ ), are important prognostic factors even beyond the initial 5 years of adjuvant endocrine therapy. Therefore, further evaluation to determine whether the LNR system can add prognostic value and complement the current nodal staging system in HR+ / HER2- patients treated with NCT is worthwhile.

Many studies have documented the usefulness of LNR; however, the thresholds used for defining low- and high-risk groups varied [11]. Vinh-Hung et al. [12], who investigated the "optimal" cut-off values for categorizing a continuous variable of LNR with minimal loss of information, analyzed the data for 1,829 women with node-positive breast cancer and identified two LNR cut-off points, 0.20 and 0.65 , which predicted breast cancer survival more adequately than $\mathrm{pN}$ categories. Compared with the $\mathrm{pN}$ classification, the LNR classification was more robust without overlapping CIs and without crossing of survival curves between the intermediate (LNR, 0.21 to 0.65 ) and high (LNR, > 0.65) risk groups. However, the cut-off points of 0.20 and 0.65 were not validated in patients treated with NCT, while the values were able to identify poor prognostic patients after primary surgery [13-15]. In our study population, LNR classification did not show an independent prognostic significance when the ypN stage was included in the multivariate model. The superiority of LNR classification over the current ypN stage was not supported by this study.

We note that our study had several limitations. First, its retrospective nature may have resulted in selection bias. To minimize this bias, however, we included consecutive patients from the database of participating institutions. Second, HER2 targeted therapy was not included in neoadjuvant treatment, but administered as adjuvant treatment because neoadjuvant trastuzumab was not reimbursed at the time of the study in Korea. Therefore, compared to other studies, the pCR rate may have been relatively lower in our patients, without impact of survival. Last, the inclusion of $\mathrm{cN} 0$ patients in the current study raised the possibility that these patients underwent inadequate axillary surgery. However, two thirds of patients $(28 / 42,66.7 \%)$ had undergone axillary LN dissection for their axillary staging.

\section{Conclusion}

In conclusion, our findings suggest that LNR is not superior to ypN stage in predicting clinical outcome of breast cancer patients after NCT. Both LNR and ypN stage had similar prognostic values after NCT. Findings from the subgroup analysis suggest that LNR might provide some additional prognostic information in HR+/HER2- patients after NCT, compared to HER2+ or TNBC patients. Further studies are needed to evaluate the optimal cut-off points and prognostic value of LNR in breast cancer patients treated with NCT. 


\section{Electronic Supplementary Material}

Supplementary materials are available at Cancer Research and Treatment website (http://www.e-crt.org).

\section{Conflicts of Interest}

Conflict of interest relevant to this article was not reported.

\section{Acknowledgments}

The authors thank the Medical Research Collaborating Center at Seoul National University Bundang Hospital for statistical analyses.

\section{Author Details}

${ }^{1}$ Division of Hematology and Medical Oncology, Department of Internal Medicine, Seoul National University Bundang Hospital, Seongnam, ${ }^{2}$ Department of Internal Medicine, Asan Medical Center, University of Ulsan College of Medicine, Seoul, ${ }^{3}$ Department of Internal Medicine, Seoul National University Hospital, Seoul, ${ }^{4}$ Department of Internal Medicine, SMG-SNU Boramae Medical Center, Seoul, ${ }^{5}$ Department of Internal Medicine, Kyungpook National University Hospital, Daegu, ${ }^{6}$ Department of Internal Medicine, Kyung Hee University Medical Center, Seoul, ${ }^{7}$ Department of Internal Medicine, Ajou University Hospital, Suwon, ${ }^{8}$ Department of Internal Medicine, Seoul St. Mary's Hospital, College of Medicine, The Catholic University of Korea, Seoul, ${ }^{9}$ Department of Internal Medicine, National Cancer Center, Goyang, ${ }^{10}$ Department of Internal Medicine, Korea University Anam Hospital, Seoul, ${ }^{11}$ Department of Internal Medicine, Severance Hospital, Yonsei University College of Medicine, Seoul, ${ }^{12}$ Department of Internal Medicine, Samsung Medical Center, Seoul, Korea

\section{References}

1. Fisher B, Bryant J, Wolmark N, Mamounas E, Brown A, Fisher ER, et al. Effect of preoperative chemotherapy on the outcome of women with operable breast cancer. J Clin Oncol. 1998; 16:2672-85.

2. Bear HD, Anderson S, Smith RE, Geyer CE Jr, Mamounas EP, Fisher B, et al. Sequential preoperative or postoperative docetaxel added to preoperative doxorubicin plus cyclophosphamide for operable breast cancer: National Surgical Adjuvant Breast and Bowel Project Protocol B-27. J Clin Oncol. 2006;24:2019-27.

3. van der Hage JA, van de Velde CJ, Julien JP, Tubiana-Hulin M, Vandervelden C, Duchateau L. Preoperative chemotherapy in primary operable breast cancer: results from the European Organization for Research and Treatment of Cancer trial 10902. J Clin Oncol. 2001;19:4224-37.

4. Cortazar P, Zhang L, Untch M, Mehta K, Costantino JP, Wolmark N, et al. Pathological complete response and longterm clinical benefit in breast cancer: the CTNeoBC pooled analysis. Lancet. 2014;384:164-72.

5. Kuerer HM, Newman LA, Buzdar AU, Hunt KK, Dhingra K, Buchholz TA, et al. Residual metastatic axillary lymph nodes following neoadjuvant chemotherapy predict disease-free survival in patients with locally advanced breast cancer. Am J Surg. 1998;176:502-9.

6. Pierga JY, Mouret E, Dieras V, Laurence V, Beuzeboc P, Dorval $\mathrm{T}$, et al. Prognostic value of persistent node involvement after neoadjuvant chemotherapy in patients with operable breast cancer. Br J Cancer. 2000;83:1480-7.
7. Symmans WF, Peintinger F, Hatzis C, Rajan R, Kuerer H, Valero $\mathrm{V}$, et al. Measurement of residual breast cancer burden to predict survival after neoadjuvant chemotherapy. J Clin Oncol. 2007;25:4414-22.

8. Colleoni M, Bagnardi V, Rotmensz N, Dellapasqua S, Viale G, Pruneri $G$, et al. A risk score to predict disease-free survival in patients not achieving a pathological complete remission after preoperative chemotherapy for breast cancer. Ann Oncol. 2009;20:1178-84.

9. Neuman H, Carey LA, Ollila DW, Livasy C, Calvo BF, Meyer AA, et al. Axillary lymph node count is lower after neoadjuvant chemotherapy. Am J Surg. 2006;191:827-9.

10. Erbes T, Orlowska-Volk M, Zur Hausen A, Rucker G, Mayer $S$, Voigt $M$, et al. Neoadjuvant chemotherapy in breast cancer significantly reduces number of yielded lymph nodes by axillary dissection. BMC Cancer. 2014;14:4.

11. Woodward WA, Vinh-Hung V, Ueno NT, Cheng YC, Royce $\mathrm{M}$, Tai $\mathrm{P}$, et al. Prognostic value of nodal ratios in node-positive breast cancer. J Clin Oncol. 2006;24:2910-6.

12. Vinh-Hung V, Verkooijen HM, Fioretta G, Neyroud-Caspar I, Rapiti E, Vlastos G, et al. Lymph node ratio as an alternative to $\mathrm{pN}$ staging in node-positive breast cancer. J Clin Oncol. 2009;27:1062-8.

13. Danko ME, Bennett KM, Zhai J, Marks JR, Olson JA Jr. Improved staging in node-positive breast cancer patients using lymph node ratio: results in 1,788 patients with longterm follow-up. J Am Coll Surg. 2010;210:797-805.

14. Ahn SH, Kim HJ, Lee JW, Gong GY, Noh DY, Yang JH, et al. 
Lymph node ratio and $\mathrm{pN}$ staging in patients with nodepositive breast cancer: a report from the Korean breast cancer society. Breast Cancer Res Treat. 2011;130:507-15.

15. Dings PJ, Elferink MA, Strobbe LJ, de Wilt JH. The prognostic value of lymph node ratio in node-positive breast cancer: a Dutch nationwide population-based study. Ann Surg Oncol. 2013;20:2607-14.

16. Liu D, Chen Y, Deng M, Xie G, Wang J, Zhang L, et al. Lymph node ratio and breast cancer prognosis: a meta-analysis. Breast Cancer. 2014;21:1-9.

17. Keam B, Im SA, Kim HJ, Oh DY, Kim JH, Lee SH, et al. Clinical significance of axillary nodal ratio in stage II/III breast cancer treated with neoadjuvant chemotherapy. Breast Cancer Res Treat. 2009;116:153-60.

18. Saxena N, Hartman M, Aziz R, Rapiti E, Bhoo Pathy N, Lim $\mathrm{SE}$, et al. Prognostic value of axillary lymph node status after neoadjuvant chemotherapy: results from a multicentre study. Eur J Cancer. 2011;47:1186-92.

19. Chen S, Liu Y, Huang L, Chen CM, Wu J, Shao ZM. Lymph node counts and ratio in axillary dissections following neoadjuvant chemotherapy for breast cancer: a better alternative to traditional pN staging. Ann Surg Oncol. 2014;21:42-50.

20. von Minckwitz G, Untch M, Blohmer JU, Costa SD, Eidtmann $\mathrm{H}$, Fasching PA, et al. Definition and impact of pathologic complete response on prognosis after neoadjuvant chemotherapy in various intrinsic breast cancer subtypes. J Clin Oncol. 2012;30:1796-804.

21. Lips EH, Mulder L, de Ronde JJ, Mandjes IA, Koolen BB, Wessels LF, et al. Breast cancer subtyping by immunohistochemistry and histological grade outperforms breast cancer intrinsic subtypes in predicting neoadjuvant chemotherapy response. Breast Cancer Res Treat. 2013;140:63-71.

22. Tausch C, Taucher S, Dubsky P, Seifert M, Reitsamer R, Kwasny $W$, et al. Prognostic value of number of removed lymph nodes, number of involved lymph nodes, and lymph node ratio in 7502 breast cancer patients enrolled onto trials of the Austrian Breast and Colorectal Cancer Study Group (ABCSG). Ann Surg Oncol. 2012;19:1808-17.

23. Goldhirsch A, Wood WC, Coates AS, Gelber RD, Thurlimann B, Senn HJ, et al. Strategies for subtypes: dealing with the diversity of breast cancer: highlights of the St. Gallen International Expert Consensus on the Primary Therapy of Early Breast Cancer 2011. Ann Oncol. 2011;22:1736-47.

24. Sestak I, Dowsett M, Zabaglo L, Lopez-Knowles E, Ferree S, Cowens JW, et al. Factors predicting late recurrence for estrogen receptor-positive breast cancer. J Natl Cancer Inst. 2013; 105:1504-11. 